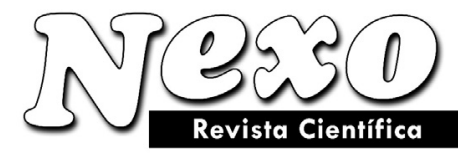

ISSN 1818-6742

Impreso en Nicaragua. www.nexo.uni.edu.ni

Vol. 23, No. 02, pp.53-61/Noviembre 2010

\title{
Gestión del manejo integral de los desechos sólidos en el hospital Alemán Nicaragüense de Managua
}

\author{
K. Blandón ${ }^{1}$, Z. Castellanos. ${ }^{2 *}$ \\ ${ }^{1}$ Centro de Investigación y Estudios del Medio Ambiente, Universidad Nacional de Ingeniería (UNI) \\ PO Box 5595, Managua, Nicaragua \\ e-mail: ciema@uni.edu.ni \\ ${ }^{2}$ Centro de Investigación de Ecosistemas Acuáticos, Universidad Centro Americana (UCA) \\ PO Box 69, Managua, Nicaragua \\ e-mail: castellanosz@ns.uca.edu.ni
}

(recibido/received: 22-Marzo-2009; aceptado/accepted: 10-Noviembre-2010)

\begin{abstract}
RESUMEN
La gestión de los desechos sólidos hospitalarios (DSH) del Hospital Alemán Nicaragüense (HAN) fue estudiada. El objetivo general propuesto fue un sistema de gestión de los desechos sólidos hospitalarios (comunes y peligrosos sin incluir los radiactivos), generados en el HAN. Se efectuó la cuantificación de los DSH durante el periodo de muestreo, elaboración de un listado de los DSH, se identificaron problemas existentes en el manejo actual de éstos desechos, y se elaboró una propuesta de plan de manejo seguro de los mismos, la que contempla: Plan integral de manejo de DSH que incluye todos los desechos (comunes y peligrosos, sin incluir los radiactivos), una propuesta de equipo adecuado para recolección y transporte interno y externo de DSH; propuesta de construcción de depósito para almacenamiento temporal interno de los DSH; y una propuesta de sistema de tratamiento y disposición final alternativo para los DSH/P generados en el HAN.
\end{abstract}

Palabras claves: desechos sólidos hospitalarios, gestión, manejo.

\begin{abstract}
The study was the management of Hospital Solid Waste (HSW) at the Nicaraguan German Hospital (NGH). The general objective proposed was a system of management of hospital waste solid waste (both non-hazardous but excluding radioactive) generated by the NGH. A quantification of the HSW was carried out during the assessment period and a list of HSW was formulated. The current problems in the handling of waste were identified and a proposal for the safe handling of waste was put forward, those under consideration are: an integrated plan for handling of HSW to include all waste (non-hazardous and hazardous but excluding radioactive) a proposal for adequate equipment for the internal and external collection and transportation of HSW. A proposal for the construction of an internal temporary storage area for HSW and a proposal for a treatment system and definitive alternative for the disposal of HSW produced by NGH.
\end{abstract}

Keywords: Hospital solid waste, management, handling.

\footnotetext{
* Autor para correspondencia
} 


\section{INTRODUCCIÓN}

Un sistema adecuado de manejo de los residuos sólidos en un centro de atención de salud, permitirá controlar y reducir con seguridad y economía los riesgos para la salud asociados con los residuos sólidos. El correcto gerenciamiento de los residuos sólidos significa no sólo controlar y disminuir los riesgos, sino lograr la minimización de los residuos desde el punto de origen, lo cual elevaría también la calidad y eficiencia de los servicios que brinde el centro de atención de salud.

En el presente estudio se realiza una propuesta de gestión integral de los desechos sólidos hospitalarios (DSH) generados en el centro de atención en salud primario Hospital Alemán Nicaragüense (HAN). Esta propuesta está basada en el diagnóstico del manejo actual de los DSH. También se abordan todas las fases del manejo de los DSH, se intenta profundizar en aquellos aspectos que son responsabilidad del personal encargado del manejo de los DSH del HAN, en la caracterización de estos desechos y en la necesidad de reducir la generación de desechos peligrosos, entre otros factores.

Finalmente, de acuerdo a los resultados obtenidos se elaboró la propuesta de un plan de manejo seguro de los DSH y sistema de tratamiento alternativo y disposición final de los DSH generados en el HAN, acorde a la situación económica del hospital. Por considerarse de vital importancia para los trabajadores vinculados con el manejo de los desechos sólidos de este centro, se propone un componente de capacitación educativo para los trabajadores y autoridades de dirección y administración dentro de la propuesta de Plan de manejo seguro de los DSH generados en el HAN, contribuyendo de ésta manera a reducir los accidentes laborales ocasionados por la falta de conciencia alrededor del tema en cuestión.

\section{METODOLOGÍA}

\section{Perfil general del área de estudio: Hospital Alemán Nicaragüense (HAN)}

El Hospital Alemán Nicaragüense (HAN), fundado el 18 de agosto de 1985, con el nombre de Hospital Carlos Marx, está ubicado en el denominado Distrito VI de la capital, en las cercanías del kilómetro 6 de la carretera norte. Brinda servicios a la zona oriental de la capital y a los municipios de Tipitapa y San Francisco Libre, pero no es raro encontrar pacientes de localidades tales como San Carlos, Bonanza y Corn Island. Con un promedio de 1,200 pacientes internos que se atienden mensualmente, la demanda sobrepasa muchas veces la capacidad del hospital, que cuenta con 230 camas. La afluencia es particularmente grande en la clínica obstétrica, donde nacen un promedio de 600 niños por mes (MINSA, 1992). En un área aproximada de $2800 \mathrm{~m}^{2}$, el Hospital Alemán Nicaragüense brinda a la población servicios de atención de emergencia, consulta externa, hospitalización, atención quirúrgica, servicios de laboratorios clínicos y radiológicos, anatomía patológica, morgue y farmacia.

\section{Técnica de muestreo}

Al realizar el estudio se tomó como muestra la cantidad total de DSH (comunes y peligrosos, sin incluir los radiactivos) producidos diariamente durante el periodo de estudio, en las diferentes áreas del HAN, los que se clasificaron y cuantificaron de acuerdo con los diferentes tipos de DSH.

Previo al muestreo de los DSH en el HAN, se estableció contacto con las autoridades correspondientes a fin de contar con su autorización y apoyo para la realización de este estudio, luego se procedió a visitar el sitio de estudio para tener una idea del manejo que actualmente se le da a los DSH en este hospital, rutas de muestreo, disposición temporal y final, etc.

Durante el muestreo, se realizó la selección de un sitio de acopio para la basura recolectada por turnos diariamente. Los criterios empleados para definir este sitio fueron: cercanía al contenedor de almacenamiento final, existencia de infraestructura para la protección de lluvia y características del piso que garantizara condiciones de seguridad e higiene durante la limpieza diaria, así como protección del agua subterránea.

La técnica de recolección de los DSH (comunes y peligrosos, sin incluir los radiactivos) consistió en acompañar al personal encargado de esta actividad a lo largo de toda la ruta establecida durante los turnos matutino y vespertino que se ejecutan de lunes a sábados; y un único turno correspondiente al día domingo. 
Los empaques utilizados durante el período de muestreo, fueron debidamente rotulados con el nombre del área $o$ sala correspondiente. Posteriormente, los desechos fueron transportados en contenedores pequeños hasta el sitio de disposición temporal.

Con el objetivo de determinar la composición física de los desechos (\%) y las densidades promedio por día ( $\mathrm{kg} /$ día), se procedió al pesado de los desechos por áreas (bolsas rotuladas), para ello se utilizó un recipiente plástico, con capacidad para 201 , el que fue previamente pesado. Este procedimiento se llevó a cabo durante todo el período de muestreo y los días de validación.

El horario de recolección y pesaje de los desechos que genera la sala de labor y parto, difiere del establecido en el párrafo anterior, esto fue debido a las particularidades de generación y tipo de desechos (placentas generadas durante el día y la noche anterior). Esta actividad se realizó a las 5:00 A.M. Por medidas de seguridad, se optó por realizar el pesaje incluyendo el recipiente metálico que almacena estos desechos en la misma sala de labor y partos. El valor obtenido fue afectado por el peso que representa el recipiente metálico.

Descripción de los procedimientos empleados para el cálculo de las variables cuantitativas

\section{a) Procedimiento para determinar el peso}

Se estableció un sitio para realizar el peso de todos los desechos sólidos generados en el centro hospitalario. Se colocó una balanza de pie en un sitio que permitió la actividad de manipuleo de las bolsas. Con el fin de establecer la forma de obtener el peso de los desechos sólidos, se utilizó un recipiente plástico con capacidad para 201 previamente pesado. El pesaje se efectuó por salas, todas las bolsas sin abrir, hasta obtener el total de desechos sólidos por turnos y salas.

b) Procedimiento para la determinación del volumen de los DSH producidos por salas

Fueron medidas la altura total y el diámetro interno del recipiente a usar. Los DSH recolectados por salas se vertieron en el recipiente y se sacudieron suavemente para eliminar los espacios vacíos y permitir que los desechos se conformaran mejor. Esta acción se realizó sin presionar los desechos. Por diferencia de volumen entre el volumen del recipiente y el volumen de los desechos, se encontró el dato de volumen de los desechos generados. La ecuación utilizada para encontrar el volumen fue:

$$
\mathrm{V}=\mathrm{h} \pi \mathrm{r}^{2}
$$

Donde:

$$
\begin{aligned}
& \mathrm{h}=\text { altura ocupada por el recipiente } \\
& \mathrm{r}=\text { radio del recipiente. }
\end{aligned}
$$

El volumen total se obtuvo al realizar la suma de los volúmenes obtenidos en cada sala, por cada día de muestreo y de validación.

c) Procedimiento para el cálculo de la densidad de los DSH

Se utilizó un recipiente de 20 lts. de capacidad y una balanza de pie con una precisión de \pm 2 gr. Se midió el peso del recipiente y se calculo el volumen del mismo. Los desechos fueron colocados dentro del depósito sin hacer presión y el mismo se agitó, de forma tal que permitiera el llenado completo de los espacios vacíos en el mismo. Posteriormente, se procedió a pesar el receptáculo con los desechos, y por la diferencia entre ambas medidas (lleno y vacio) se determinó el peso neto de los desechos sólidos. Con el peso y el volumen, fue posible calcular las densidades de estos dividiendo su peso en kilogramos entre su respectivo volumen en metros cúbicos.

Descripción de los procedimientos empleados para el cálculo de las variables cualitativas

a) Procedimiento para determinar la composición física de los DSH

Del total de bolsas pesadas con desechos sólidos y previamente clasificadas por salas, se seleccionó al azar una por sala. Fue tomada una muestra de aproximadamente $0.5 \mathrm{~m} 3$, la que fue vertida sobre la superficie del embaldosado, formando un cumulo. Los desechos de cartón y madera, fueron reducidos, hasta obtener un tamaño aproximado de $15 \times 15 \mathrm{~cm}$. Posteriormente la muestra fue homogenizada, mezclándola en su totalidad. A continuación la muestra fue dividida en cuatro partes y de estas se escogieron dos partes opuestas para la formación de una muestra más pequeña que fuese representativa. La muestra menor, se mezcló y dividió nuevamente en cuatro partes, luego, se escogieron dos partes opuestas y se 
formó otra submuestra más pequeña. Esta operación se repitió hasta obtener un ejemplar de aproximadamente $50 \mathrm{~kg}$ de desechos. Una vez finalizado este proceso, se realizó la separación de los desechos por tipos (plástico, madera, aluminio, telas, vidrios, orgánicos, etc.). Cada tipo de desecho clasificado fue pesado directamente en el recipiente plástico con capacidad para 201 . Se calculode forma porcentual (\%) a cada tipo de desecho. Esta actividad fue realizada con la mayor rapidez posible, con la finalidad de conservar las características particulares de humedad de cada uno de los residuos.

Procedimiento utilizado para la determinación de las restantes variables cualitativas

Para estudiar las prácticas habituales aplicadas en el manejo de los DSH del centro hospitalario, sus técnicas de recolección y transporte, así como su situación higiénica sanitaria, se realizó un análisis basado en la gestión operativa interna que posee el centro.

Procedimiento para la elaboración del plan de manejo seguro de los DSH

Fue elaborado un Plan de Manejo Seguro de los DSH, el cual se fundamento en la metodología propuesta por Gutiérrez et al. (2004). La metodología fue ajustada al hospital siguiendo los siguientes pasos: A partir de los resultados del análisis de los DSH, y de la evaluación de la gestión operativa interna del centro hospitalario, utilizada por los mismos al momento de la realización de este estudio, fueron definidos los objetivos generales y específicos que se proyecta conseguir mediante la ejecución del plan. A partir de los objetivos, se eligieron las estrategias para lograr el cumplimiento de estos, de igual forma se identificaron los aspectos de la gestión actual del HAN, los cuales permitieron detectar diversos aspectos a ser mejorados, también se establecieron las pautas a seguir para ello con la finalidad de dar cumplimiento a los objetivos establecidos. Para cada una de las alternativas identificadas se elaboró un subprograma con objetivos, metas e indicadores de verificación. Además se determinaron las acciones necesarias para el desarrollo de cada subprograma. Finalmente, se diseñó una pequeña estrategia de implementación, evaluación y seguimiento del plan integral de manejo de los DSH.
Propuesta del sistema de tratamiento y disposición final alternativo para los DSH/P generados en el HAN

La propuesta del sistema de tratamiento y disposición final alternativo de los DSH/P fue realizado basándose en los sistemas propuestos por el Centro Panamericano de Ingeniería Sanitaria, de la Organización Panamericana de la Salud (CEPIS/OPS), teniendo en consideración la existencia del sistema de tratamiento por incineración que posee el HAN.

Análisis estadístico de los datos obtenidos

Para el análisis estadístico fueron elaboradas matrices de datos para el estudio de la información obtenida. Estos datos fueron examinados mediante el uso de la estadística descriptiva. Los resultados fueron presentaron mediante tablas y figuras (histogramas y diagramas de pastel). El paquete estadístico utilizado fue el Microsoft Excel.

\section{RESULTADOS Y DISCUSIÓN}

Análisis cuantitativo de los DSH ( $\mathrm{kg} /$ día) producidos en el Hospital Alemán Nicaragüense (HAN) durante el periodo de estudio.

La cantidad total de DSH producidos en el HAN durante los siete días de muestreo fue de $2,497.95 \mathrm{~kg}$ con una producción promedio total $356.85 \mathrm{~kg} /$ día. La mayor producción de desechos sólidos se originó el día lunes con un total de $516.36 \mathrm{~kg}$ /día, y corresponden a los dos turnos (matutino y vespertino) de todas las salas, mientras que la menor producción se originó el día domingo con un total de $218.41 \mathrm{~kg} /$ día, generados solamente por el turno matutino. La mayor producción registrada el día lunes es debida a la acumulación que se produce del día anterior (domingo), esto es debido a que en los fines de semana solamente se realiza un turno de recolección. La menor cantidad producida el día domingo, se debió a que hay menor afluencia de pacientes al centro durante el fin de semana, y a que prácticamente solo se atiende las emergencias.

En relación a la producción de desechos semanales, la sala de atención a pacientes que produjo mayor cantidad fue el quirófano y cirugía con $598.18 \mathrm{~kg}$, dicha sala es la que generó la mayor cantidad de desechos diariamente, por otro lado, y según los cálculos realizados, se pudo observar que la cocina del centro hospitalario es el área que registra una mayor producción de desechos por dia, 
y por ende de forma semanal. Los desechos generados en este sitio (no es sala de atención a pacientes), son considerados inocuos cuando estos no se mezclan con el resto de desecho, aunque los mismos pueden volverse peligrosos al momento de la recolección, esto debido a que tales desechos son depositados juntos en un mismo contenedor, el cual contiene bolsas con desechos peligrosos procedentes de otras salas, p.ej., desechos del quirófano, ginecología, cirugía, pediatría, etc.

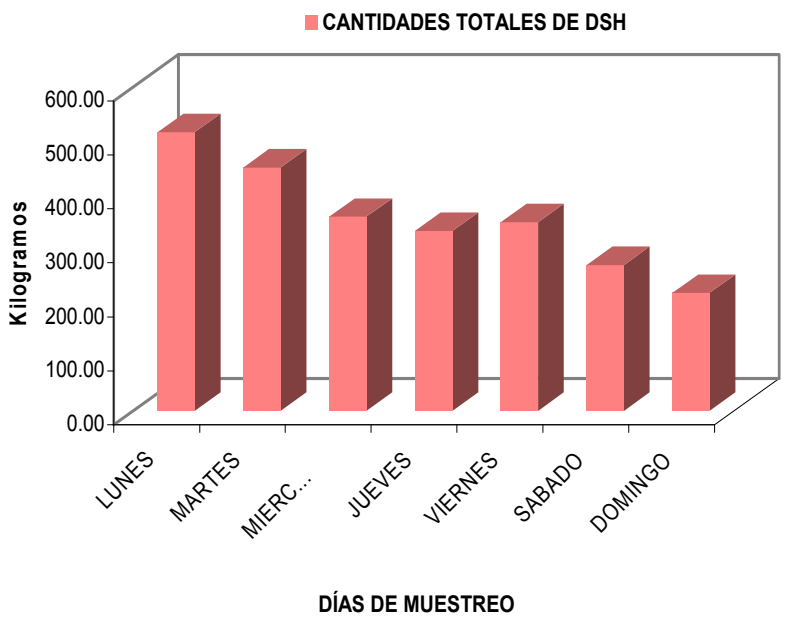

Figura 1.Cantidades totales (kg/día) de DSH producidos en el HAN.

La Figura 1, muestra que el día lunes se generó la mayor cantidad de desechos sólidos en el centro hospitalario, también se puede observar que entre los días miércoles, jueves y viernes, la producción de DSH es aproximadamente similar, y, con tendencia a descender durante el fin de semana, principalmente el día domingo, donde se observó la menor producción de desechos en comparación con el resto de los días.

\section{Listado de los tipos de DSH que se generarán el HAN}

De acuerdo a la composición física general de los tipos de desechos y en base a lo observado durante la fase de muestreo y validación, los tipos de DSH que se producen en el hospital bajo estudio fueron los siguientes:

a) Plásticos: Bolsas y recipientes plásticos de diferentes productos de limpieza y alimenticios, principalmente botellas (jugos, refrescos, agua), y envases de medicamentos tales como bolsas de suero, de sangre, sondas, etc. b) Tela: Restos de ropas de pacientes y otras piezas desechadas propiedad del hospital tales como: sábanas y gabachas. Partes utilizadas de gasas y vendas.

c) Papel: desechos de papelería de oficinas, cartón, empaques de medicamentos, papel encerado y papel higiénico.

d) Aluminio: Latas de jugos y gaseosas.

e) Orgánico: desechos de jardinería, cocina y de cafeterías.

f) Vidrio: Envases de medicamentos (inyecciones, frascos de jarabes), botellas de jugos y gaseosas.

g) Cortopunzantes: Bisturís, agujas y vidrio.

h) Restos patológicos: restos humanos (placentas y partes humanas).

i) Otros: Cuero (restos de zapatos y fajas), apósitos (toallas sanitarias, restos de algodón).

Composición física de los tipos de DSH (kg/dia) producidos en el hospital bajo estudio.

Los componentes más abundantes fueron los corto punzantes con un total de $104.54 \mathrm{~kg}$; cabe destacar que este tipo de desechos no se recolectan a diario, sino que solo dos veces por semana (lunes y viernes), por lo que no son comparables con el resto de componentes. En un segundo lugar, los restos de origen patológico representaron un total $75.23 \mathrm{~kg}$, seguidos de los desechos orgánicos que alcanzaron la cifra de $25.91 \mathrm{~kg}$ en los siete días de muestreo. Cantidades menores se correspondieron a los desechos de vidrio, aluminio y tela, con $0.71 \mathrm{~kg}, 2.15 \mathrm{~kg}$ y $9.37 \mathrm{~kg}$ respectivamente. La Fig. 2 muestra la composición de los DSH que se generados en el HAN.

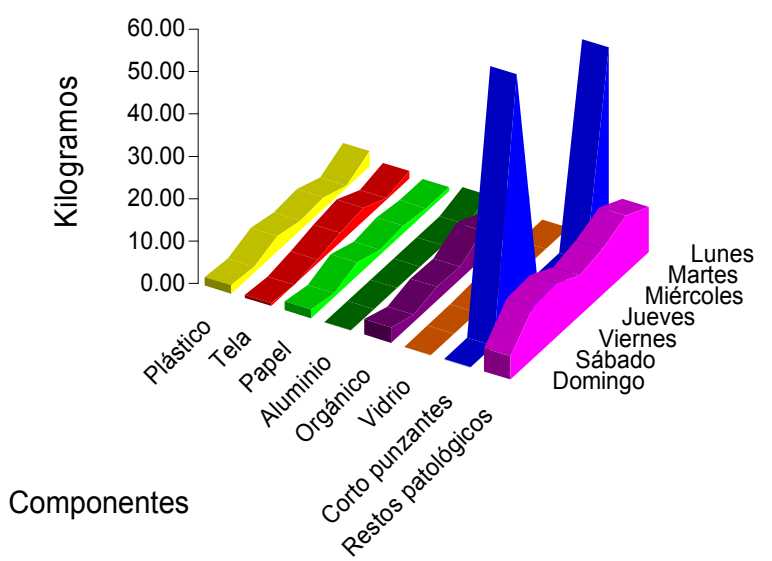

Figura 2. Composición física de los tipos de DSH ( $\mathrm{kg} /$ día) producidos en el HAN 
Composición física porcentual (\%) de los DSH durante el periodo de muestreo

El porcentaje promedio de los componentes sólidos hospitalarios producidos en el HAN es presentada en la Figura 3. Tal y como puede observarse, el mayor porcentaje se correspondió a los restos patológicos (44.48\%), seguido de los desechos clasificados como cortopunzantes $(19.61 \%)$. Los componentes con menor porcentaje promedio fueron el aluminio $(0.58 \%)$, y el vidrio $(0.51 \%)$. Cabe destacar que debido a que los desechos del grupo cortopunzantes solamente se recolectaron dos veces por semana durante el periodo de muestreo, no fueron comparados con el resto de componentes.

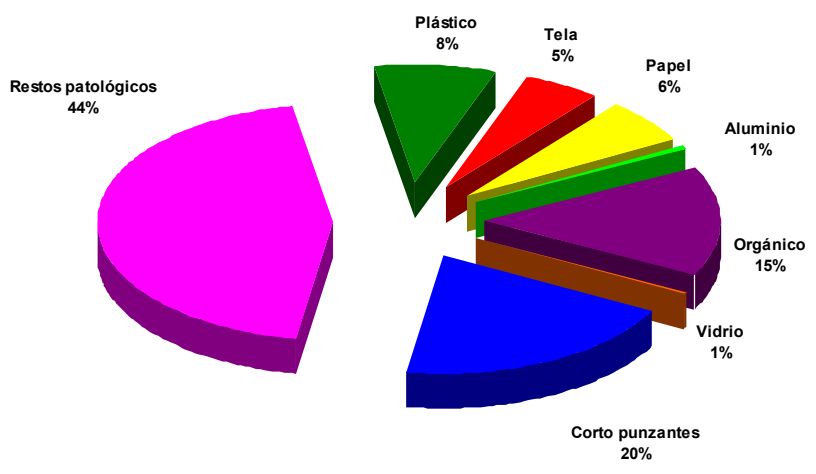

Figura 3. Composición física de los DSH expresada en porcentaje

Densidad $(\mathrm{kg} / \mathrm{m} 3)$ promedio/día de los DSH generados en el HAN

La densidad promedio por día de los DSH encontrada en el Hospital Alemán Nicaragüense fue variable. Los desechos sólidos hospitalarios con mayor densidad promedio por día fueron los patológicos, los cuales alcanzaron un valor de $315.47 \mathrm{~kg} / \mathrm{m} 3$. La cantidad de restos de vidrio encontrada fue mínima, por lo que al expresarla en términos de densidad, ésta se representó con el valor de $0.00 \mathrm{~kg} / \mathrm{m} 3$. Se entiende que esta situación es debido a que el vidrio por ser objetos corto punzantes son segregados de forma separada de las salas donde se producen, y que los pocos restos encontrados junto con los demás desechos sólidos fueron puestos por descuido o de forma accidental en los contenedores de desechos comunes.

El segundo tipo de residuos con menos densidad promedio fue el aluminio con $5.97 \mathrm{~kg} / \mathrm{m} 3$, en este tipo de residuos lo que más se encontró fueron latas de jugos y bebidas gaseosas, las que en su mayoría son producidas por los visitantes y personal del hospital, más que por los pacientes.

Durante este periodo, la densidad promedio por día de los desechos sólidos hospitalarios, fue de $509.51 \mathrm{~kg} / \mathrm{m} 3$. En la Fig. 4 se observa la diferencia entre la densidad promedio por día por cada tipo de desecho sólido hospitalario observado durante el periodo de muestreo.

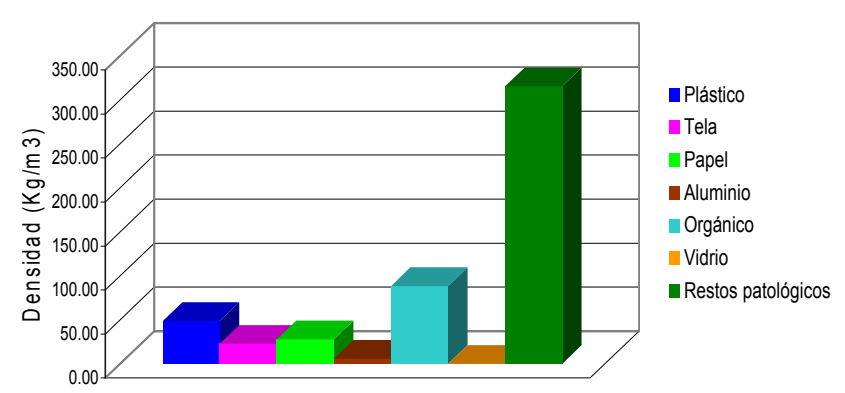

Figura 4. Densidad (kg/m3) promedio/día de los componentes de los DSH generados en el HAN

Gestión operativa interna de los DSH en el Hospital Alemán Nicaraguense

El hospital estudiado posee su propio Sistema de Gestión Operativa Interna de DSH, el que está a cargo del Subcomité de Desechos Sólidos Hospitalarios, de éste depende el área de servicios generales a través del Departamento de Ornato y Limpieza. Este comité hace grandes esfuerzos por manejar adecuadamente los DSH que se producen a diario en el hospital, pero enfrentan muchos problemas con relación a la gestión operativa interna de los desechos sólidos hospitalarios los que se resumen principalmente: la falta de cumplimiento de las normas de bioseguridad por parte del personal encargado de la recolección de los DSH, y la inexistente e inadecuada segregación de los mismos. Adicionalmente, se encontró que existe escaso apoyo económico por parte del Ministerio de Salud, ya que enfrentan muchas limitaciones financieras, finalmente se conoció de una irregularidad en la periodicidad de recolección de los DSH por parte de la Alcaldía de Managua, que ocasiona la acumulación de los desechos en el sitio de almacenamiento terciario.

El HAN posee un incinerador para el tratamiento de los desechos patológicos; a los cortopunzantes se les aplica como sistema de tratamiento, la incineración, misma que 
no es efectiva ya que no se logra un reducción significativa de los mismos.

Propuesta de plan de manejo integral y sistema de tratamiento alternativo y disposición final de los DSH generados en el HAN

Ante los problemas y las debilidades encontradas en la gestión operativa interna del HAN, se propuso un plan de manejo seguro de los DSH, el que incluye: Un plan integral de manejo de Desechos Sólidos Hospitalarios el cual incluye tanto a los comunes como a los peligrosos (excluyendo los desechos radiactivos); una propuesta de equipamiento adecuado para la recolección, y para el transporte interno y externo de los desechos; una propuesta de construcción de un depósito para el almacenamiento temporal interno de los DSH del centro hospitalario; y una propuesta de sistema de tratamiento y disposición final alternativo para los DSH/P generados en el HAN

Considerando que el Hospital Alemán Nicaragüense ya cuenta con un sistema de tratamiento para sus desechos sólidos peligrosos, como es el sistema de incineración, la propuesta del sistema de tratamiento y disposición final para los DSH peligrosos, se limita a un procedimiento alternativo en caso de que el incinerador deje de funcionar por alguna situación imprevista (o programada). El sistema de tratamiento y disposición final alternativa propuesta es la construcción de un Relleno de Emergencia para el hospital, el cual es un método provisional, y es adecuado tanto para desechos infecciosos (restos patológicos), como para objetos cortopunzantes. El método actualmente utilizado en el hospital de incinerar de forma artesanal los objetos cortopunzantes ha demostrado que no es efectivo para la reducción de estos, ya que su incineración es parcial, y la mayoría de los casos los mismos quedan intactos, por lo que en esta propuesta se establece que los desechos tales como agujas, bisturí y todos aquellos residuos cortopunzantes reciban un tratamiento especial antes de ser depositados en el relleno de emergencia; por el peligro que representan para el personal que los maneja, $y$ en el caso de que estos puedan ser desenterrados por animales o por personas ajenas al hospital, se propone como sistema de tratamiento el Encapsulado, esta propuesta es una opción simple, económica, segura y viable para el HAN, este método también puede aplicarse a productos farmacéuticos. Para los DSH comunes, se propone la construcción de un relleno sanitario mecanizado.

\section{CONCLUSIONES}

El Hospital Alemán Nicaragüense (HAN) cuenta dentro de su Sistema organizacional, con un Subcomité de Desechos Sólidos, el que desarrolla un Sistema de Gestión Operativa Interna de DSH, mismo que funciona con muchas limitantes, principalmente financieras.

No ha logrado llevar a cado el cumplimiento del Plan de Manejo Integral de los DSH, el cual tiene contemplado un componente de capacitación. El grado de conciencia organizacional apreciada en el personal del hospital es relativamente bajo. Se observó que el personal encargado de la recolección interna de los DSH, a veces no usa sus equipos de protección personal, aunque estén concientes del riesgo que corren con la manipulación inadecuada de estos desechos.

Durante el estudio se encontró que el manejo de los DSH del HAN en términos generales no es objeto de una vigilancia sistemática que garantice un control efectivo del mismo. Los desechos son recolectados con algún grado de precaución únicamente durante el manejo interno, obviando su tratamiento y disposición final.

Los tipos de DSH que se generan en el HAN son: plásticos, telas, papel, aluminio, orgánicos, vidrio, cortopunzantes y restos patológicos. En términos de cuantificación, son los desechos patológicos y los orgánicos los que representan el mayor porcentaje, mientras que los desechos que en menor porcentaje se generan, son el vidrio y aluminio.

Los resultados de la generación de DSH en el HAN son elevados $(2,497.95 \mathrm{Kg})$, si se comparan con los obtenidos de un estudio realizado en el año 1999, en los hospitales Berta Calderón, Lenin Fonseca y Manolo Morales, y en los que se cuantificaron producciones de DSH de $1,690.5 \mathrm{~kg}, 1,854.98 \mathrm{~kg}$ y $1,994.97 \mathrm{~kg}$, respectivamente, de igual forma este dato sigue siendo mayor en relación al reportado por el Hospital Militar Alejandro Dávila Bolaños en 1999, donde se obtuvo un total de $1,929.99 \mathrm{~kg}$.

En general, existe una serie de problemas alrededor del manejo seguro de los desechos sólidos en el HAN: No existe una segregación adecuada de los desechos peligrosos con los comunes, los restos de cocina se mezclan con los desechos de las áreas de atención a 
pacientes. Los únicos desechos que no se mezclaban con el resto, son los desechos patológicos, específicamente las placentas.

Aunque se utilizan bolsas plásticas en los recipientes contenedores de desechos sólidos en las salas de generación, estas bolsas son de un solo color, por lo que los desechos sólidos se depositan en ellas sin separación de ningún tipo. Durante el transporte interno se produce una mezcla de desechos peligrosos con comunes, y estos no son transportados de manera adecuada y segura al sitio de almacenamiento temporal interna del HAN.

El sistema de disposición final utilizado con los desechos patológicos antes de adquirir el incinerador era enterrarlos. Pero se pudo observar que esta disposición final, era realizaba de una forma muy deficiente, creándose por tanto el riesgo de ocasionar problemas ambientales y propagación de vectores.

El sistema de tratamiento que se aplicaba a los desechos cortopunzantes era la incineración, pero con ello no se lograba una destrucción completa de los mismos. Según se pudo constatar en el sitio, una vez instalado y funcionando el incinerador, la práctica de quemar los restos cortopunzantes continuaba siendo el sistema de tratamiento empleado para tales restos.

El sitio actual de almacenamiento temporal interno, no presta las condiciones adecuadas ya que se constato que no cumple con los requerimientos mínimos recomendados por los organismos internacionales tales como la OPS/OMS y CEPIS. En las condiciones presentes, este sitio es propicio para la propagación de vectores, y para la contaminación ambiental al encontrarse al aire libre y ser de fácil acceso para los animales y personas.

Existe deficiencia por parte de la Alcaldía de Managua en cuanto a la recolección de los desechos sólidos en éste hospital, lo que ocasiona acumulación de los mismos, propiciando aún más la proliferación de vectores y dándole un mal aspecto al hospital.

Se recomienda de vital importancia la separación de los DSH, peligrosos del resto por parte del personal del hospital, para brindarles un manejo adecuado y seguro, de acuerdo con las normas de bioseguridad nacionales e internacionales establecidas.
Se propone la adquisición de un terreno en las afueras de Managua para la construcción de un relleno sanitario mecanizado exclusivo para los DSH, además de la adquisición de dos camiones contenedores refrigerados por parte del MINSA o del HAN para el transporte seguro de los DSH hasta éste relleno.

El Plan de manejo integral de los DSH, el sistema de tratamiento alternativo y disposición final para los DSH (comunes y peligrosos, sin incluir los radiactivos) propuesto en este estudio, tiene como objetivos mejorar los aspectos administrativos, técnicos y operativos del sistema actual, reducir la generación de desechos sólidos hospitalarios y dar un manejo integral a éstos.

Tanto las autoridades superiores del HAN como el Subcomité de Manejo de los Desechos Sólidos hacen grandes esfuerzos por realizar una adecuada gestión de los desechos sólidos que se generan en el hospital, pero los problemas económicos y la falta de apoyo por parte del MINSA hacen que su labor se vea disminuida, no lográndose alcanzar totalmente los objetivos que puedan plantearse en pro del manejo adecuado de los DSH.

\section{REFERENCIAS}

Chang M. A. (1995). Manejo y Tratamiento de Residuos Sólidos Hospitalarios. p. 55 Informe Técnico Final. Perú.

Gutiérrez Z. A y Pereira C. S. (2004). Diseño de un Plan Integral de Manejo de Residuos Sólidos en la Universidad Centroamericana. Tesis para optar el Título de Ingeniero en Calidad Ambiental. p. 135 Universidad Centroamericana. Facultad de Ciencia, Tecnología y Ambiente. Managua, Nicaragua.

López H. S. (2003). Diagnóstico y diseño de rehabilitación sistema hidrosanitario, contra incendios y pluvial del Hospital Alemán Nicaragüense- MINSA. Managua, Nicaragua.

Ministerio del Ambiente y los Recursos Naturales (MARENA) (2004). Bases de la Política Nacional Sobre Gestión Integral de los Residuos Sólidos 2003-2004. p.30, Versión final. Managua, Nicaragua.

Ministerio de Salud (MINSA). (2003). Ley General de Salud, Ley No. 423 y su Reglamento. p. 65La Gaceta, Diario Oficial. Managua, Nicaragua. 
MINFIN, MINSA, OPS, OMS. (1992). Resultados preliminares sobre el estado actual de los hospitales de Managua. p. 16, Managua, Nicaragua.

Ministerio de Salud (MINSA). (1992). Hospital "Carlos Marx" en Managua: Sinónimo de buena atención en salud. p. 36 Managua, Nicaragua.

Ministerio de Salud (MINSA). (1989). Disposiciones Sanitarias y Reglamento de Inspección Ciudadana. La Gaceta, p. 16, Diario Oficial. Managua, Nicaragua.

Ministerio de Salud (MINSA). (1980). Reglamento General de Hospitales. p. 91, Managua, Nicaragua.

Ministerio de Salud. (1998). Tecnología de tratamiento de residuos sólidos en establecimientos de salud. p. 63, Programa de Fortalecimiento de Servicios de Salud, Área de Residuos Sólidos Hospitalarios. Lima, Perú.

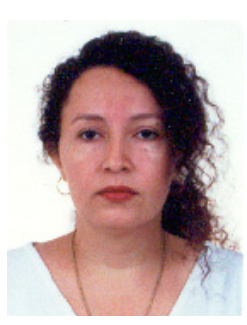

Karla Jacqueline Blandón Rivera. Master en Ingeniería Ambiental, graduada en el Centro de Investigación y Estudios en Medio Ambiente de la Universidad Nacional de Ingeniería (CIEMA-UNI) en el año 2006. Ingeniero Civil, graduada en Universidad Nacional de Ingeniería (UNI-RUPAP) en 1994. Experiencia en supervisión y construcción de obras verticales y horizontales.

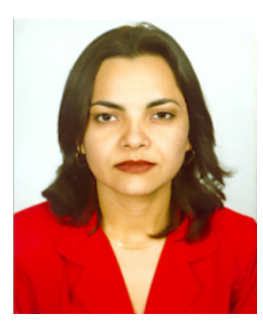

Zunilda del Carmen Castellanos Corrales. Master en Ciencias Ambientales, graduada en el Centro de Investigación y Estudios en Medio Ambiente de la Universidad Nacional de Ingeniería (CIEMA-UNI) en el año 2006. Licenciada en Ecología y Recursos Naturales graduada en la Universidad Centroamericana (UCA) en 1998. Cargo desempeñado actualmente: Responsable del Área de Histopatología y responsable de Control de Calidad del Laboratorio del Centro de Investigación de Ecosistemas (CIDEA-UCA). 